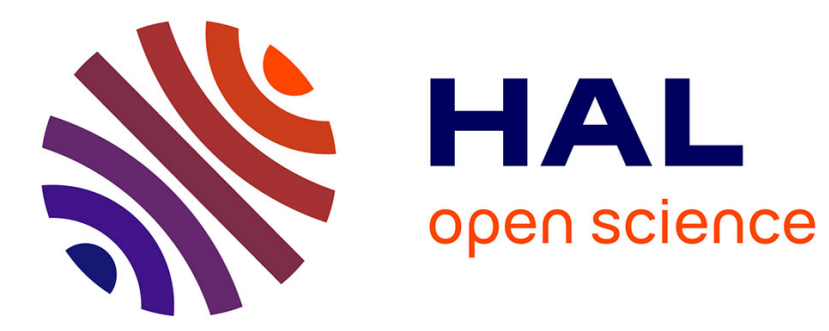

\title{
Permanent Magnet Synchronous Motor control via Parameter Dependent Relay Control
}

\author{
Romain Delpoux, Laurentiu Hetel, Alexandre Kruszewski
}

\section{To cite this version:}

Romain Delpoux, Laurentiu Hetel, Alexandre Kruszewski. Permanent Magnet Synchronous Motor control via Parameter Dependent Relay Control. American Control Conference (ACC), Jun 2014, Portland, United States. 10.1109/ACC.2014.6858935 . hal-00957800

\section{HAL Id: hal-00957800 \\ https://hal.science/hal-00957800}

Submitted on 11 Mar 2014

HAL is a multi-disciplinary open access archive for the deposit and dissemination of scientific research documents, whether they are published or not. The documents may come from teaching and research institutions in France or abroad, or from public or private research centers.
L'archive ouverte pluridisciplinaire HAL, est destinée au dépôt et à la diffusion de documents scientifiques de niveau recherche, publiés ou non, émanant des établissements d'enseignement et de recherche français ou étrangers, des laboratoires publics ou privés. 


\title{
Permanent Magnet Synchronous Motor control via Parameter Dependent Relay Control
}

\author{
Romain Delpoux, Laurentiu Hetel and Alexandre Kruszewski
}

\begin{abstract}
The article presents a novel control strategy for the control of Permanent Magnet Synchronous Motor (PMSM). The approach is motivated by the fact that PMSM are usually controlled by relays and thus only a finite set of control inputs is available. However in classical control design the use of Pulse-Width Modulation (PWM) ignores the relay nature of the actuators. Here we propose a direct relay control. As PMSM may be modeled as Linear Parameter Varying (LPV) systems, we propose a Parameter Dependent Relay (PDR) control. A design based on Linear Matrix Inequalities (LMI) allows to derive the switching surfaces, which depend on the motor position. The theory described is illustrated by simulations results.
\end{abstract}

Index Terms-PMSM, relay feedback control, linear matrix inequalities, switched systems.

\section{INTRODUCTION}

Permanent Magnet Synchronous Motors (PMSM's) are widely used in the industry. They are more reliable than brush Direct Current (DC) motors and produce a higher torque per volume. From a control engineer perspective, using the field oriented reference frame, referred to as $d-q$ frame, offers several advantages since it provides a simplified structure for the control, by avoiding sinusoidal functions. The control laws in the $d-q$ frame are referred as Field Oriented Control (FOC). They represent the most used control schemes in the field. Several control methodologies have been proposed in the literature : input-output linearization [4], flatness based approach [20] or sliding mode control [23], [17], [6]. However most of the existing control laws are usually implemented via Pulse-Width Modulation (PWM) hardware and ignore the relay nature of the actuators. Here we propose a direct relay control which may use the advantages of the switching actuators in power electronics.

Relay feedback was proposed in variable structure systems [21], [8], [9], [22] and has very interesting robustness properties faced to matched perturbations. In this field, several problems remain unsolved, such as choosing the switching surfaces to optimize system performances, the robustness properties or the size of the domain of attraction.

R. Delpoux is with Laboratoire des Sciences de l'Information et des Sytèmes (CNRS UMR 7296) INSM team, 8, Bd Louis XIV, 59046 Lille Cedex and Non-A Team, Inria Lille Nord Europe, France romain. delpoux@ensam.eu

L. Hetel and A. Kruszewski are with Laboratoire d'Automatique, Génie Informatique et Signal (CNRS UMR 8219), Ecole Centrale de Lille, 59651 Villeneuve d'Ascq cedex, France laurentiu.heteldec-lille.fr and alexandre.kruszewskidec-lille.fr

The research leading to these results has received funding from the European Community's 7th Framework Program (FP7/2007-2013) (grant agreement No 257462) HYCON2 Network of Execellence.
Recently, Linear Matrix Inequalities (LMI) conditions have been proposed for designing a relay control in the case of Linear Time Invariant (LTI) systems with time varying sampling [12]. The method considers LMI techniques used for systems with saturation [5], [13], [14], [15] and convex optimization techniques [18]. Up to now, it exists few switching surfaces optimization tools applied to nonlinear systems. Here we extend the results from [12] to the case of Linear Time-Varying systems [3], [2], [11] which are interesting since they may be used for approximating locally nonlinear systems. The main contribution is to propose an LMI based approach for the design of switching surfaces which are parameterized by time varying parameters. The objective is to find a Parameter Dependent Relay (PDR) controlling directly the commutations of the actuators. When applied to the case of nonlinear systems, the method leads to designing nonlinear switching surfaces guaranteeing the stability of the system under actuator constraints. An illustration of the theory through simulation results using real motor parameters is proposed.

The paper is structured as follow: Section II describes the LPV model of the PMSM for the purpose of relay control. Section III is dedicated to the generic design of the PDR control for LPV systems. The simulation results on the PMSM are proposed in Section IV.

Notations: The identity (or null) matrix with appropriate dimension is denoted by $\mathbf{I}$ (or $\mathbf{0}$ ). For a square symmetric matrix, $M>0(M<0)$ indicates that $M$ is positive (negative) definite. For a full rank square symmetric matrix $M, M^{-1}$ denotes the inverse of $M$. For a symmetric matrix,

$$
M=\left[\begin{array}{ll}
A & B \\
* & C
\end{array}\right] .
$$

where $*$ denotes an element that may be inferred by symmetry.

We denote by $\mathcal{I}_{N}$ the set $\{1,2, \ldots, N\}$.

The symbol $\Delta$ denotes the unit simplex in $\mathbb{R}^{N}$ defined by:

$$
\Delta=\left\{\mu \in \mathbb{R}^{N}: \mu_{i} \geq 0, i \in \mathcal{I}_{N}, \sum_{i=1}^{N} \mu_{i}(t)=1\right\} .
$$

For a given set $\mathcal{S} \in \mathbb{R}^{n}$ the symbol $\overline{\operatorname{conv}}\{\mathcal{S}\}$ denotes the closed convex hull of the set. For a symmetric positive definite matrix $P \in \mathbb{R}^{n \times n}$ and a positive scalar $c$ we denote by $\mathcal{E}(P, c)$ the ellipsoid:

$$
\mathcal{E}(P, c)=\left\{x \in \mathbb{R}^{n}: x^{T} P x<c\right\} .
$$

Let $\mathcal{B}(x, c)$ denotes the open ball centered on $x \in \mathbb{R}^{n}$ with 
radius $c>0$ :

$$
\mathcal{B}(x, c)=\left\{y \in \mathbb{R}^{n}:|x-y|<c\right\} .
$$

Given a constant scalar $U>0$, we define the finite set:

$$
\Psi_{m}(U)=\{-U, U\}^{m} .
$$

Given a continuous function $f: \mathbb{R}^{n} \rightarrow \mathbb{R}$ and a compact set $\mathcal{S}$ we denote by:

$$
\underset{s \in \mathcal{S}}{\operatorname{argmin}} f(s)=\{s \in \mathcal{S}: f(s) \leq f(r), \forall r \in \mathcal{S}\} .
$$

For a set $\mathcal{S} \subset \mathbb{R}^{m}, K \in \mathbb{R}^{m \times n}$, we denote:

$$
\mathcal{C}_{\mathcal{S}}(K)=\left\{x \in \mathbb{R}^{n}: K x \in \overline{\operatorname{conv}}\{\mathcal{S}\}\right\}
$$

\section{Motivation}

The equations (6) give the standard PMSM model in the phase (or winding) variables:

$$
\left\{\begin{aligned}
L \frac{d i_{\alpha}}{d t}= & v_{\alpha}-R i_{\alpha}+K \Omega \sin \left(n_{p} \theta\right), \\
L \frac{d i_{\beta}}{d t}= & v_{\beta}-R i_{\beta}-K \Omega \cos \left(n_{p} \theta\right), \\
J \frac{d \Omega}{d t}= & K\left(i_{\beta} \cos \left(n_{p} \theta\right)-i_{\alpha} \sin \left(n_{p} \theta\right)\right) \\
& -f_{v} \Omega-\tau,
\end{aligned}\right.
$$

where $v_{\alpha}$ and $v_{\beta}$ are the voltages applied to the two phases of the PMSM, $i_{\alpha}$ and $i_{\beta}$ are the two phase currents, $L$ is the inductance of a phase winding, $R$ is the resistance of a phase winding, $K$ is the back-EMF constant (and also the torque constant), $\theta$ is the angular position of the rotor, $\Omega=d \theta / d t$ is the angular velocity of the rotor, $n_{p}$ is the number of pole pairs (or rotor teeth), $J$ is the moment of inertia of the rotor (including the load), $f_{v}$ is the coefficient of viscous friction and $\tau$ represents the load torque.

The non-linear state space representation of the system of equations (6) is given by:

$$
\dot{x}_{\alpha \beta}(t)=f\left(x_{\alpha \beta}, t\right)+B v_{\alpha \beta}(t)+D \varpi(t),
$$

where $x_{\alpha \beta}^{T}=\left[\begin{array}{lll}i_{\alpha} & i_{\beta} & \Omega\end{array}\right], v_{\alpha \beta}^{T}=\left[\begin{array}{ll}v_{\alpha} & v_{\beta}\end{array}\right]$ and $\varpi=\tau$. The function $f\left(x_{\alpha \beta}, t\right)$ is defined by:

$$
\begin{gathered}
f\left(x_{\alpha \beta}\right)=\left[\begin{array}{c}
-\frac{R}{L} i_{\alpha}+\frac{K}{L} \Omega \sin \left(n_{p} \theta\right) \\
-\frac{R}{L} i_{\beta}-\frac{K}{L} \Omega \cos \left(n_{p} \theta\right) \\
\frac{K}{J}\left(i_{\beta} \cos \left(n_{p} \theta\right)-i_{\alpha} \sin \left(n_{p} \theta\right)\right)-\frac{f_{v}}{J} \Omega
\end{array}\right], \\
B=\left[\begin{array}{cc}
\frac{1}{L} & 0 \\
0 & \frac{1}{L} \\
0 & 0
\end{array}\right] \text { and } D=\left[\begin{array}{c}
0 \\
0 \\
\frac{1}{J}
\end{array}\right] .
\end{gathered}
$$

Considering that each motor phase is actuated via commutation, the control vector $v_{\alpha \beta}$ may take values in a finite set defined by:

$$
\Psi_{2}(V)=\left\{v_{\alpha \beta} \in \mathbb{R}^{2}: v_{\alpha}, v_{\beta} \in\{-V, V\}\right\},
$$

where $V$ represents the maximal voltage that the control can deliver.

\section{Model in the rotating frame $(d-q)$}

In the phases frame the signals $i_{\alpha}$ and $i_{\beta}$ vary at $n_{p}$ times the frequency of rotation. This high frequency problem is alleviated by the use of the direct quadrature $(d-q)$ transformation. This transformation changes the frame of reference from the fixed phase axes to axes moving with the rotor. Equation (8) gives the transformation performed to obtain the rotating frame:

$$
R(\theta)=\left[\begin{array}{cc}
\cos \left(n_{p} \theta\right) & \sin \left(n_{p} \theta\right) \\
-\sin \left(n_{p} \theta\right) & \cos \left(n_{p} \theta\right)
\end{array}\right] .
$$

The variables in the rotating frame are expressed by:

$$
\left[\begin{array}{c}
i_{d} \\
i_{q}
\end{array}\right]=R(\theta)\left[\begin{array}{l}
i_{\alpha} \\
i_{\beta}
\end{array}\right]
$$

and

$$
\left[\begin{array}{l}
v_{d} \\
v_{q}
\end{array}\right]=R(\theta)\left[\begin{array}{l}
v_{\alpha} \\
v_{\beta}
\end{array}\right] .
$$

The state space representation is then given by:

$$
\dot{x}_{d q}(t)=A_{d q}(\Omega(t)) x_{d q}(t)+B v_{d q}(t)+D \varpi(t),
$$

where $x_{d q}^{T}=\left[\begin{array}{lll}i_{d} & i_{q} & \Omega\end{array}\right], v_{d q}^{T}=\left[\begin{array}{ll}v_{d} & v_{q}\end{array}\right]$, and:

$$
A_{d q}(\Omega(t))=\left[\begin{array}{ccc}
-\frac{R}{L} & n_{p} \Omega(t) & 0 \\
-n_{p} \Omega(t) & -\frac{R}{L} & -\frac{K}{L} \\
0 & \frac{K}{J} & -\frac{f_{v}}{J}
\end{array}\right] .
$$

The matrices $B$ and $D$ remain unchanged. Consider that $\Omega(t)$ ranges between known extremal values $\Omega(t) \in[\underline{\Omega}, \bar{\Omega}]$. In this frame the PMSM can be described using a LPV state space representation. The state space representation of the system depends linearly on a vector of time varying parameters: $\Omega(t), \sin \left(n_{p} \theta(t)\right), \cos \left(n_{p} \theta(t)\right)$. The model may be represented as follows:

$$
\left\{\begin{aligned}
\dot{x}_{d q}(t)= & A(\mu(t)) x_{d q}(t)+B v_{d q}(t)+D \varpi(t), \\
A(\mu(t))= & \sum_{i=1}^{N_{A}} \mu_{i}(t) A_{i}, \quad \forall i, \mu_{i}(t) \geq 0 \\
& \sum_{i} \mu_{i}(t)=1
\end{aligned}\right.
$$

where $N_{A}=2$, with $A \in\left[A_{d q}(\underline{\Omega}), A_{d q}(\bar{\Omega})\right]$, and $v_{d, q}(t)$ are defined such that $\forall \theta \in[0,2 \pi]$. The control is defined by:

$$
v_{d q}(t)=k\left(x_{d q}(t), \theta(t)\right)
$$

where:

$$
k: \mathbb{R}^{3} \times \mathbb{R} \rightarrow \bar{v}_{d q}(\theta) .
$$




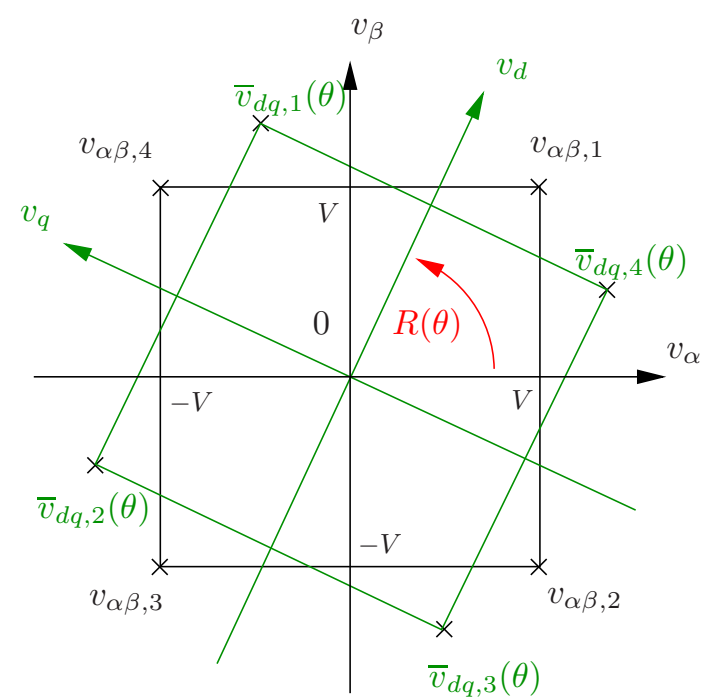

Fig. 1. Finite set of control in the fixe frame and in the rotating frame.

The control vector $v_{d q}(t)$ is a PDR control which takes values in a finite set $\bar{v}_{d q}(\theta)$ defined by:

$$
\bar{v}_{d q}(\theta)=\left\{v_{d q} \in \mathbb{R}^{2}: v_{d q}=R(\theta) v_{\alpha \beta}, v_{\alpha \beta} \in \Psi_{2}(V)\right\}
$$

For a given $V$, the objective is to determine the switching surfaces in the state space which ensure the closed loop stability of the system (12) with the control law (13). The control inputs in the different frames are represented Fig. 1.

\section{PARAMETER DEPENDENT RELAY (PDR) CONTROL}

We consider the class of Linear Parameter-Varying (LPV) systems with the state-space realization:

$$
\dot{x}=A(\mu(t)) x+B(\mu(t)) u,
$$

where $x \in \mathbb{R}^{n}$ is the state vector and $u \in \mathbb{R}^{m}$ is the control vector, the matrices $A \in \mathbb{R}^{n \times n}, B \in \mathbb{R}^{n \times m}$, are polytopic matrices with the following form:

$$
A(\mu(t))=\sum_{i=1}^{N} \mu_{i}(t) A_{i}, B(\mu(t))=\sum_{i=1}^{N} \mu_{i}(t) B_{i}
$$

with $A_{1}, \ldots, A_{N}, B_{1}, \ldots, B_{N}$ being known constant matrices. The vector $\mu(t)=\left[\begin{array}{lll}\mu_{1}(t) & \ldots & \mu_{N}(t)\end{array}\right]^{T}$ is a vector of real and known parameters which evolves in the unit simplex $\Delta$.

We assume that for each $\mu \in \Delta$ the control $u$ may only takes values in a finite set which depends on the parameter $\mu$. We define this set of finite values $\mathcal{V}_{\mu}$ by:

$$
\mathcal{V}_{\mu}=\left\{v_{i}(\mu(t)), i \in \mathcal{I}_{k}\right\}, v_{i}: \Delta \rightarrow \mathbb{R}^{m}, \forall i \in \mathcal{I}_{k}
$$

We assume that $\overline{\operatorname{conv}}\left\{\mathcal{V}_{\mu}\right\}$ is a non empty set containing the origin in its interior for any $\mu \in \Delta$.

The objective is to find a PDR control $u(x, \mu)$ which locally stabilize the system (16):

$$
u: \mathbb{R}^{m} \times \Delta \rightarrow \mathcal{V}_{\mu}
$$

The vector set $\mathcal{V}_{\mu}$ is a known set corresponding to the actuators constraints.

Given that $u$ is discontinuous, the closed loop vector field is discontinuous. Therefore system solutions are considered in the sense of Filippov [10]. We recall the definition of locally asymptotically stability. One poses:

$$
\dot{x}=f(t, x),
$$

where $f(t, x)=A(\mu(t)) x+B(\mu(t)) u$.

Definition 1: [1] The equilibrium point $x=0$ of (20) is

- stable if, for each $\epsilon>0$, there is $\delta=\delta\left(\epsilon, t_{0}\right)>0$ such that:

$$
\left\|x\left(t_{0}\right)\right\|<\delta \Rightarrow\|x(t)\|<\epsilon, \forall t \geq t_{0} \geq 0,
$$

- locally asymptotically stable at the origin if it is stable and there is $c=c\left(t_{0}\right)>0$ such that $x(t) \rightarrow 0$ as $t \rightarrow \infty$, for all $\left\|x\left(t_{0}\right)\right\|<c$.

\section{A. Stabilization by PDR control}

In this paragraph we show how classical LMIs [5] may be used to find a control law under the form (19), that is a PDR control.

Proposition 1: Consider system (16) with the description (17). Assume that the set of LMI conditions:

$$
Q A_{i}^{T}+A_{i} Q+B_{i} Y+Y^{T} B_{i}^{T}<-2 \delta Q, i=i, \ldots, N
$$

hold with $Q=Q^{T}>0, Y \in \mathbb{R}^{m \times n}$ and $\delta>0$. Then there exists a positive $\gamma$ such that the function $V(x)=x^{T} Q^{-1} x$, satisfies:

$$
\begin{aligned}
& \frac{\partial V}{\partial x}(A(\mu) x+B(\mu) u(x, \mu))<-2 \delta V(x), \\
& \forall x \in \Gamma \backslash\{0\}, \forall \mu \in \Delta,
\end{aligned}
$$

where:

$$
u(x, \mu) \in \underset{v \in \mathcal{V}_{\mu}}{\operatorname{argmin}} x^{T} Q^{-1} B(\mu) v, x \in \Gamma \backslash\{0\},
$$

with:

$$
\Gamma=\mathcal{E}\left(Q^{-1}, \gamma\right)
$$

Proof: It is desired to design the PDR control (24) that ensures locally the stabilization. The condition (22) means that the Lyapunov function $V(x)=x^{T} Q^{-1} x$ satisfies:

$$
\begin{aligned}
& 2 x^{T} Q^{-1}\left(\left(A(\mu)+B(\mu) Y Q^{-1}\right) x\right)<-2 \delta V(x), \\
& \forall x \neq 0, \forall \mu \in \Delta,
\end{aligned}
$$

i.e. it is a Lyapunov function for the system (16) with the state-feedback control law $Y Q^{-1} x$.

Since for all $\mu \in \Delta, \overline{\operatorname{conv}}\left\{\mathcal{V}_{\mu}\right\}$ is non empty and contains the origin in its interior, there exists a positive scalar $\gamma$ such that:

$$
\forall x \in \mathcal{E}\left(Q^{-1}, \gamma\right), \mu \in \Delta \Rightarrow Y Q^{-1} x \in \overline{\operatorname{conv}}\left\{\mathcal{V}_{\mu}\right\}
$$


one has:

$$
\mathcal{C}_{\mathcal{V}_{\mu}}\left(Y Q^{-1}\right):\left\{x \in \mathbb{R}^{n}: Y Q^{-1} x \in \overline{\operatorname{conv}}\left\{\mathcal{V}_{\mu}\right\}\right.
$$

Let us denote $\Gamma:=\mathcal{E}\left(Q^{-1}, \gamma\right)$. Then for any $x \in \Gamma$ and $\mu \in \Delta$ there exist $k$ scalars $\alpha_{j}(x, \mu) \geq 0, j \in \mathcal{I}_{k}$, with $\sum_{j=1}^{k} \alpha_{j}(x, \mu)=1$ such that:

$$
Y Q^{-1} x=\sum_{j=1}^{k} \alpha_{j}(x, \mu) v_{j}(\mu) .
$$

From (25) and (28) we have:

$$
\begin{aligned}
& \sum_{j=1}^{k} \alpha_{j}(x, \mu) 2 x^{T} Q^{-1}\left(A(\mu) x+B(\mu) v_{j}(\mu)\right) \\
& <-2 \delta V(x), \forall \mu \in \Delta \text { and } \forall x \in \Gamma \backslash\{0\} .
\end{aligned}
$$

Considering $\alpha_{j}(x, \mu)>0, \mu \in \Delta$ and $j \in \mathcal{I}_{k}$, there must be at least one $j \in \mathcal{I}_{k}$ such that:

$$
2 x^{T} Q^{-1}\left(A(\mu) x+B(\mu) v_{j}(\mu)\right)<-2 \delta V(x),
$$

$\forall x \in \Gamma \backslash\{0\}, \forall \mu \in \Delta$.

Since $\Gamma$ represents a sub-level set of $V(x)$, local stabilization in $\Gamma$ with a finite control set is ensured by choosing the control $u(x, \mu)$ with the steepest descend of the Lyapunov function:

$$
u(x, \mu) \in \underset{v \in \mathcal{V}_{\mu}}{\operatorname{argmin}} x^{T} Q^{-1} B(\mu) v .
$$

Since all minimizers ensure the decay of $\mathrm{V}$ and the switching surfaces are continuous the the stability (in the sense of Filipov solutions) is insured.

\section{B. Characterization of the domain of attraction}

In the previous section we have shown how to guarantee the local stability of the system (16) with the PDR control law (24) in an ellipsoid $\Gamma$. In practice it is often useful to provide an estimation of this domain of attraction.

Since $\mu \in \mathcal{V}_{\mu}, \overline{\operatorname{conv}}\left\{\mathcal{V}_{\mu}\right\}$ is non empty and contains the origin, remark that there exists a polytopic region:

$$
\begin{aligned}
\mathcal{Q} & =\overline{\operatorname{conv}}\left\{q_{1}, q_{2}, \ldots, q_{p}\right\} \\
& =\left\{y \in \mathbb{R}^{m}: h_{i} y \leq 1, i \in \mathcal{I}_{N_{h}}\right\},
\end{aligned}
$$

such that:

$$
\mathcal{Q} \in \overline{\operatorname{conv}}\left\{\mathcal{V}_{\mu}\right\}, \forall \mu \in \Delta \text { and } 0 \in \operatorname{Int}\{\mathcal{Q}\} .
$$

Using the polytope $\mathcal{Q}$ one can adjust the design condition (22) so to include an optimization of the domain of attraction.

Proposition 2: Consider system (16) and assume that there exists $Q=Q^{T}>0, Y$ and a positive scalar $\delta$ such that:

$$
Q A_{i}^{T}+A_{i} Q+B_{i} Y+Y^{T} B_{i}^{T}<-2 \delta Q, i \in \mathcal{I}_{N},
$$

$$
\begin{gathered}
{\left[\begin{array}{cc}
1 & h_{i} Y \\
* & Q
\end{array}\right]>0, i \in \mathcal{I}_{N_{h}},} \\
{\left[\begin{array}{ll}
e I & I \\
* & Q
\end{array}\right]>0 .}
\end{gathered}
$$

Then the equilibrium point $x=0$ of the closed-loop system (16)-(24) is locally asymptotically stable. An estimation of the domain of attraction is provided by the ball $\mathcal{B}(0, \sqrt{\epsilon})$ with $\epsilon=\frac{1}{e}$.

Proof: The objective is to find $Q$ and $Y$ such that:

$$
\forall x \in \mathcal{E}\left(Q^{-1}, 1\right) \Rightarrow Y Q^{-1} x \in \mathcal{Q} .
$$

Using Lemma 1 ([13], see appendix), (34) leads to:

$$
\begin{aligned}
1 & \leq \min _{h_{i} K x=1} x^{T} Q^{-1} x \\
& =\min _{i \in \mathcal{I}_{N_{h}}}\left(h_{i} Y Q^{-1} Q\left(Y Q^{-1}\right)^{T}\left(h_{i}\right)^{T}\right)^{-1},
\end{aligned}
$$

which may be characterized by the sufficient set of LMI

$$
\left[\begin{array}{cc}
1 & h_{i} Y \\
* & Q
\end{array}\right]>0, i \in \mathcal{I}_{N_{h}}
$$

To guarantee that the ball $\mathcal{B}(0, \sqrt{\epsilon})$ is included in the domain of decay $\mathcal{E}\left(Q^{-1}, 1\right)$ of the Lyapunov function we add the constraints [14], [13]:

$$
\left[\begin{array}{cc}
e I & I \\
* & Q
\end{array}\right]>0 .
$$

with $e=\frac{1}{\epsilon}$.

Remark ${ }^{\epsilon}$ : The feasibility of the LMI optimization problem (33) with some matrix $Q$, guarantees that any LPV system (16) with the control (24) originating from the invariant ellipsoid $\mathcal{E}\left(Q^{-1}, 1\right)$ is converging to the origin with a decay rate $\delta$. By minimizing $e$, the size of the invariant ellipsoid is maximized, in the sense that it contains the ball $\mathcal{B}(0, \sqrt{\epsilon})$ with the maximum radius $\sqrt{\epsilon}$. This allows to provide an inner ellipsoid approximation of the domain of attraction.

\section{SIMULATION RESULTS}

In this section we propose simulation results to illustrate the PDR control, applied to a PMSM. For this purpose, we design a velocity tracking control where we consider that only four control inputs are available. In this article, the control design is considered with the assumption that there is no external torque (i.e. $\tau=0$ ).

The objective is to follow a velocity reference $y_{\text {ref }}$. Given that the plant has no natural integrator, an integral action is implemented [19], to ensure tracking performance. The integral action is given by:

$$
\dot{\zeta}=y-y_{\text {ref }}=C x-y_{\text {ref }},
$$

where $\zeta$ is the output of the integrator $(\zeta(0)=0)$. A combination of the state space representation (11) and the 
integral action (38) without torque can be re-written as:

$$
\underbrace{\left[\begin{array}{c}
\dot{x}_{d q} \\
\dot{\zeta}
\end{array}\right]}_{\dot{z}}=\underbrace{\left[\begin{array}{cc}
A_{d q}(\Omega) & 0 \\
C & 0
\end{array}\right]}_{\bar{A}(\Omega)} \underbrace{\left[\begin{array}{c}
x_{d q} \\
\zeta
\end{array}\right]}_{z}+\underbrace{\left[\begin{array}{c}
B \\
0
\end{array}\right]}_{\bar{B}} u-\underbrace{\left[\begin{array}{c}
0 \\
I
\end{array}\right]}_{R} y_{r e f} .
$$

The objective is to design an asymptotically stable system such that at steady state one has $\dot{\zeta}=0$, and get the output at steady state $y=y_{\infty}$. Moreover, at steady state, one has:

$$
\dot{z}_{\infty}=\bar{A}(\Omega) z_{\infty}+\bar{B} u_{\infty}-R y_{r e f, \infty},
$$

Considering a constant reference, $y_{\text {ref }}=y_{\text {ref }, \infty}$ for $t>0$. Define

$$
\begin{aligned}
& e=z-z_{\infty}, \\
& u_{c}=u-u_{\infty} .
\end{aligned}
$$

The dynamic error equation $\dot{e}=\dot{z}-\dot{z}_{\infty}$ is defined by:

$$
\dot{e}=\bar{A}(\Omega) e+\bar{B} u_{c},
$$

where $u_{c}$ is a state feedback control law defined by:

$$
u_{c}=Y Q^{-1} e,
$$

with $Q=Q^{T}>0$ and $Y \in \mathbb{R}^{2 \times 4}$. The parameter $\Omega$ takes values in $[0,30]$ (in rad.s $s^{-1}$ ). For each $\theta$, the control $u$ is constrained to switch among four different values in the set $\left\{\mathbb{R}(\theta) \rho, \rho \in \Psi_{2}(V)\right\}$. The matrix $R(\theta)$ is defined by equation (8). For the simulations we consider the real parameters of the stepper motor bench developed in LAGIS at École Centrale de Lille [7]. The parameters are $L=9 \mathrm{mH}$, $R=3.01 \Omega, K=0.27 N . m . A^{-1}$ and $J=3 \cdot 18 \cdot 10^{-4} \mathrm{~kg} \cdot \mathrm{m}^{2}$. The number of pole pairs is $n_{p}=50$. The power supply provides a maximum voltage $v_{\max }=20 \mathrm{~V}$. The sampling period for this simulation is constant and equal to $10^{-4} \mathrm{~s}$. The relay value $V$ is equal to $v_{\max }=20 \mathrm{~V}$ leading to a set of two input each taking values in $\{-V, V\}$.

Considering as bounded time varying parameter $\sin (\theta(t))$, $\cos (\theta(t))$ and $\Omega(t)$, the system (41) may be rewritten as an LPV system of the form (16) defined by:

$$
\dot{x}=A(\mu) x+B u,
$$

with 8 vertices.

The control $u$ takes values in the finite set (18) defined by:

$$
\mathcal{V}_{\mu}=\left\{v_{i}(\mu), i \in 1, \ldots, 4\right\}=\left\{R(\theta) \rho, \rho \in \Psi_{2}(V)\right\} .
$$

In order to optimize the domain of attraction, it is necessary to find a polytopic region $\mathcal{Q} \in \mathcal{V}_{\mu}$. Note that the input $u$ takes values in the vertices of a square of size $2 \times V$ submitted to a rotation defined by $R(\theta)$. The region containing $u$ is the inscribed disc of radius $V$. This disc can be approximated by the polytope $\mathcal{Q}$ represented for which the vertices $q_{i}$ are given by:

$$
q_{i+1}=V\left[\begin{array}{c}
\cos \left(\frac{2 i \pi}{p}\right) \\
\sin \left(\frac{2 i \pi}{p}\right)
\end{array}\right], i=0, \ldots, p-1 .
$$

Each face of the polytope can be characterized by its normal:

$$
h_{i+1}=\frac{q_{i}+q_{i+1}}{1+\cos \left(\frac{2 \pi}{p}\right)}, i=0, \ldots, p-1 .
$$

The simulation are realized using Matlab/Simulink and the LMI are solved using the SeDuMi solver [16]. In order to approximate the inscribed disc by the polytope $\mathcal{Q}$ we take $p=30$. Choosing a decay rate $\delta=1$, the LMI solver computes the following $Q$ and $Y$ matrices:

$$
\begin{gathered}
Q=\left[\begin{array}{cccc}
29.6 & -4.8 & 9.4 & -0.012 \\
-4.8 & 26.6 & -15.9 & 0.038 \\
9.4 & -16.0 & 208.4 & -2.8 \\
-0.012 & 0.038 & -2.8 & 0.069
\end{array}\right], \\
Y=\left[\begin{array}{llll}
-41.7 & -39.1 & 178.5 & -0.052 \\
-56.3 & -51.2 & -74.2 & -0.59
\end{array}\right] .
\end{gathered}
$$

The $Q$ matrix defines the PDR control (24) and thus the switching regions. To illustrate the theoretical results, one proposes to compare the continuous state feedback (CSF) control law $u_{c}=Y Q^{-1} e$ with the PDR control.

Fig. 2 represents the simulation results when no external torque is added. The velocity tracking is accurate in both cases: it shows that at steady state the desired trajectory is tracked with a precision of $0.1 \mathrm{rad} . \mathrm{s}^{-1}$ for the PDR control. It must be noted that chattering phenomena appears in the PDR case leading to a tracking error slightly higher. However in this case only four control inputs are used for control. In order to show the robustness of the PDR control, Fig. 3 shows the same tracking but, in this case, an external torque is added from $t=7 \mathrm{~s}$ until the end of the simulations. Due to the control saturation, the CSF is not able to track the desired
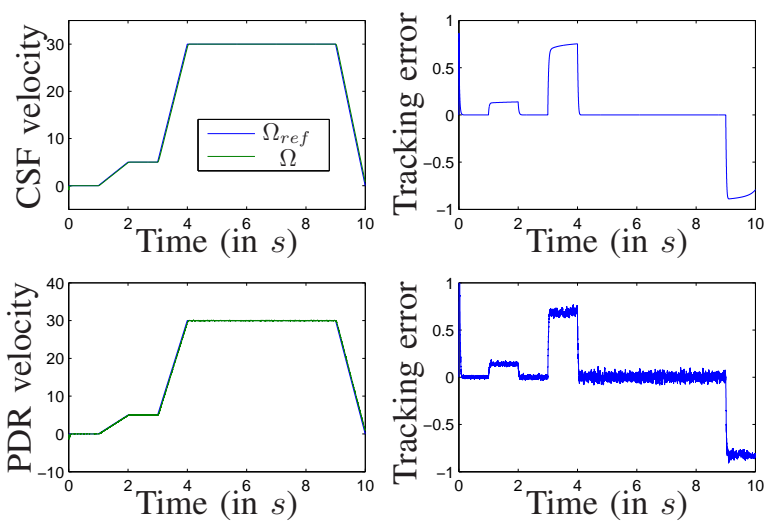

Fig. 2. CSF and PDR velocity tracking simulation without perturbation (in rad.s ${ }^{-1}$ ). 

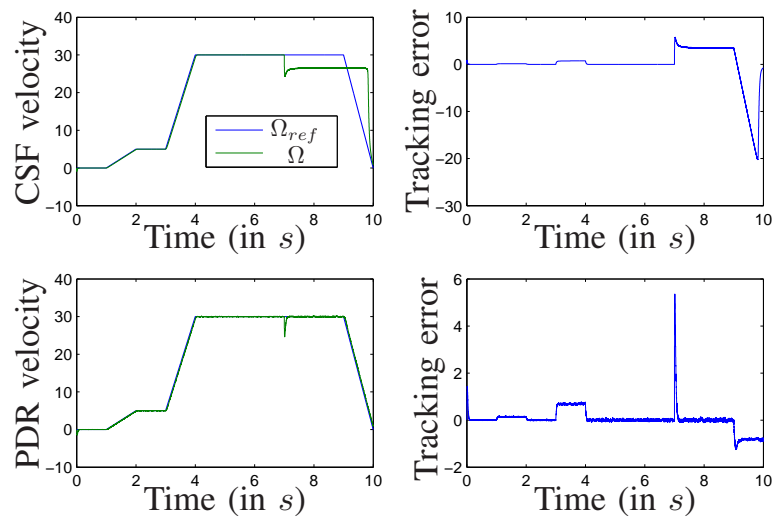

Fig. 3. CSF and PDR velocity tracking simulation with additive external torque (in rad.s ${ }^{-1}$ ).

velocity while the PDR shows good robustness properties.

\section{CONCLUSION}

This article has presented a novel control strategy for the control of PMSM. While classical control design using PWM ignores the relay nature of the actuators, in this paper a direct relay control has been considered. Based on an LPV modeling of the PMSM we have proposed a PDR control. LMI have allowed to derive the switching surfaces, which depend on the motor position. Simulation results have been presented based on real PMSM parameters and have shown that, it was possible to control a PMSM using simply the available inputs of the hardware. Moreover the simulations have pointed out the interesting robustness properties of relay control. As future work it would be interesting to consider the perturbations for the control design. A generalization to a larger class of non-linear system is under consideration. Experimental results are also in progress.

\section{APPENDIX}

Lemma 1: ([13]). Let $V(x)=x^{T} Q^{-1} x$, where $Q=$ $Q^{T}>0, \mathrm{C}$ be a row vector in $\mathbb{R}^{n}$ and $\mathrm{r}$ be a nonzero scalar. Then the minimum of $V$ along the hyperplane $\{x \mid C x=r\}$ is given by:

$$
\alpha_{r}=\frac{r^{2}}{C Q C^{T}} \text {. }
$$

\section{REFERENCES}

[1] A. Bacciotti and L. Rosier. Liapunov Functions and Stability in Control Theory. Communications and Control Engineering. Springer, 2005.

[2] G.-I. Bara, J. Daafouz, F. Kratz, and J. Ragot. Parameter-dependent state observer design for affine LPV systems. International Journal of Control, 74(16):1601-1611, 2001.

[3] J.-M. Biannic, P. Apkarian, and W.-L. Garrard. Parameter varying control of a high performance aircraft. Journal of guidance, control, and dynamics, 20:225-231, 1996.

[4] M. Bodson, J.-N. Chiasson, R.-T. Novotnak, and R.-B. Rekowski. High performance nonlinear feedback control of a permanent magnet stepper motor. IEEE Transactions on Control Systems Technology, 1(1):5-14, 1993.

[5] S. Boyd, L. El-Ghaoui, E. Feron, and V. Balakrishnan. Linear Matrix Inequalities in System and Control Theory. Studies in Applied Mathematics. SIAM, Philadelphia, PA, 1994.
[6] M. Defoort, F. Nollet, T. Floquet, and W. Perruquetti. A Third-Order Sliding-Mode Controller for a Stepper Motor. IEEE Transactions on Industrial Electronics, 56(9):3337-3346, 2009.

[7] R. Delpoux, M. Bodson, and T. Floquet. Parameter estimation of permanent magnet stepper motors without mechanical sensors. Control Engineering Practice, 26:178-187, May 2014.

[8] C. Edwards and S.-K. Spurgeon. Sliding mode control - Theory and applications. Taylor \& Francis, 1998.

[9] S.-V. Emel'yanov. Variable Structure Automatic Control Systems. Nauka, Moscou, 1967.

[10] A.-F. Filippov and F.-M. Arscott. Differential Equations with Discontinuous Righthand Sides: Control Systems. Mathematics and its Applications. Springer, 1988.

[11] J.-C. Geromel and P. Colaneri. Robust stability of time varying polytopic systems. Systems \& Control Letters, 55(1):81-85, 2006.

[12] L. Hetel, E. Fridman, and T. Floquet. Sampled-data control of LTI systems with relay: a convex optimization approach. In 9th IFAC Symposium on Nonlinear Control Systems, page to appear, Toulouse, France, 2013.

[13] H. Hindi and S. Boyd. Analysis of Linear Systems with Saturation using Convex Optimization. In 37th IEEE Conference on Decision and Control, pages 903-908, 1998.

[14] T. Hu and Z. Lin. Control Systems With Actuator Saturation: Analysis and Design. Control Engineering. Birkhäuser, springer edition, 2001.

[15] T. Hu, Z. Lin, and B.-M. Chen. Analysis and design for discretetime linear systems subject to actuator saturation. Systems \& Control Letters, 45(2):97-112, February 2002.

[16] Y. Labit, D. Peaucelle, and D. Henrion. SeDuMi Interface 1.02: a Tool for Solving LMI Problems with SeDuMi. In IEEE International Symposium on Computer Aided Control System Design, pages 272277, 2002.

[17] S. Laghrouche, F. Plestan, A. Glumineau, and R. Boisliveau. Robust second order sliding mode control for a permanent magnet synchronous motor. In 2003 American Control Conference, volume 5, pages 4071 - 4076, Denver, Colorado USA, 2003.

[18] D. Liberzon. Switching in Systems and Control. Systems \& control. Systems \& Control: Foundations \& Applications. Birkhäuser, 2003.

[19] K. Ogata. Modern Control Engineering. Instrumentation and controls series. Prentice Hall, 2010.

[20] H. Sira-Ramírez. A Passivity plus Flatness Controller for Permanent Magnet Stepper Motor. Asian Journal of Control, 2(1):1-9, 2000.

[21] Y.-Z. Tsypkin. Relay Control Systems. Nauka, Moscow, 1984.

[22] V.-I. Utkin. Sliding modes in control optimization. Communication and Control Engineering, Birkhäuser, 1992.

[23] M. Zribi, H. Sira-Ramírez, and A. Ngai. Static and dynamic sliding mode control schemes for a permanent magnet stepper motor. International Journal of Control, 74(2):103-117, 2001. 